\title{
On Resonant Tunnelling in the Biased Double Delta-Barrier
}

\author{
I. YANETKA \\ Department of Physics, Faculty of Civil Engineering, Slovak University of Technology \\ Radlinského 11, 81368 Bratislava, Slovakia
}

(Received July 10, 2009; in final form October 12, 2009)

\begin{abstract}
The solution of the one-dimensional Schrödinger wave equation is presented for the potential-energy function that describes a double delta-barrier under the application of a constant electrical field perpendicular to it. The transfer matrix technique is employed to determine the transmission coefficient in an analytical form. Some attributes of the transmission coefficient are established. The transmission coefficient is shown to exhibit maxima and minima, the conditions for maxima and minima in the transmission coefficient are discussed. The current-voltage characteristic of the biased double delta-barrier is calculated numerically. It is found to exhibit the same oscillatory behaviour as the transmission coefficient when the voltage applied to the double delta-barrier is increased. The width of the double delta-barrier is shown to modulate the peak-to-valley ratio in the current-voltage characteristic.
\end{abstract}

PACS numbers: 73.40.Gk, 03.65.-w

\section{Introduction}

This paper presents a very simple method for evaluating the tunnelling current through a sandwich structure $\mathrm{A}-\mathrm{B}-\mathrm{A}-\mathrm{B}-\mathrm{A}$ that is under the application of a constant electrical field perpendicular to its interfaces ( $\mathrm{B}$ is a very narrow single crystalline layer embedded in and being lattice-matched on both its sides with a bulk crystal A). The numerical values used in the calculation correspond to the sandwich structure $\mathrm{GaAs} / \mathrm{Al}_{1-x} \mathrm{Ga}_{x} \mathrm{As}$ made of GaAs as a bulk crystal $\mathrm{A}$ and of $\mathrm{Al}_{1-x} \mathrm{Ga}_{x} \mathrm{As}$ as a crystalline layer B. The GaAs $/ \mathrm{Al}_{1-x} \mathrm{Ga}_{x} \mathrm{As}$ system is the most known sandwich structure owing to the relative easiness of its fabrication as well as its close lattice matching [1-3].

Neglecting space charge effects and adopting the Wannier one-band approximation [4], one can diagrammatically represent the biased sandwich structure A-B-A-B-A with the flat-band scheme shown in Fig. 1 (the horizontal full lines in Fig. 1 correspond to the lower boundary of the conduction band in the regions $\mathrm{A}$ and $\mathrm{B}$ ). Thus, the narrow layers $\mathrm{B}$, which are embedded in the bulk crystal A, are modelled by one-dimensional rectangular potential barriers of a width $w_{0}$ and a height $U_{0}$; vide e.g. [5-7]. The applied constant electrical field, which is perpendicular to the interfaces of the sandwich structure, is simulated by lowering the bottom of the conduction band in the biased regions A. Thus, there is established a one-dimensional rectangular potential well of a finite width $2 a$ and a finite depth $\mathrm{eV} / 2$ in the central region $\mathrm{A}$, and a one-dimensional rectangular potential well of an infinite extent and a finite depth $\mathrm{eV}$ in that region $\mathrm{A}$, in which the electrical potential is higher by the applied voltage $V$. Here $e$ is the elementary charge and $2 a$ is the width of the central region $\mathrm{A}$. Therefore, in each region $\mathrm{A}$, the potential energy of a conduction electron is replaced by its average value. In the case of a very narrow layer $\mathrm{B}$, i.e. when $w_{0} \ll 2 a$, one can formally let the width of the rectangular potential barrier tend to zero and simultaneously let its height tend to infinity whilst keeping their product to be constant. Then, the potential-energy function that represents the rectangular potential barrier in Fig. 1 becomes a delta-function whose strength $g$ is equal to the product of the width and the height of the original rectangular potential barrier, i.e. $g=w_{0} U_{0}$. Although, the delta-function is a very simplified form of the original potential-energy function, it still enables one to get a proper insight into the transmissions through the high and narrow barrier structures [8-11]. This is also the reason why the delta-function potentials are often employed throughout many parts of the solid-state physics as a very convenient approximation to more structured and therefore more difficult, short-ranged potentials. Therefore, it must also be interesting for one to calculate the tunnelling current through the biased double delta-function potential, i.e. through the biased double delta-barrier. As far as the author knows such a calculation has not been carried out, though the transmission coefficient for the unbiased double delta-barrier is available, e.g. in [9-11].

Evidently, the use of the delta-barrier instead of the rectangular barrier is justified only when electrons trans- 


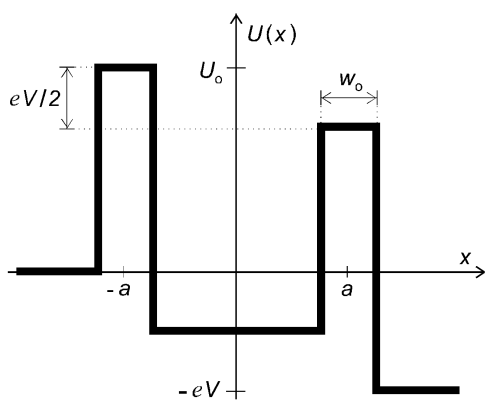

Fig. 1. Schematic diagram of the electron potential energy in the rectangular double-barrier with the voltage $V$ applied to it.

mitting through the barrier move in all the three regions A near the bottom of the conduction band. Thus, the longitudinal energy $E_{x}$ of the transmitting electron (the energy that is associated with the electron motion in the direction perpendicular to the potential barrier) should be much smaller than the height of the original rectangular potential barrier $U_{0}$, i.e. $E_{x} \ll U_{0}$. Also the applied voltage $V$ should be very small, i.e. $e V \ll U_{0}$. The width $w_{0}$ of the original rectangular potential barrier (the width of the layer B) should also be much smaller than the reduced longitudinal wavelength $\lambda$ of the transmitting electron. Thus, it must be valid, $w_{0} \ll \lambda=\hbar / \sqrt{2 m E_{x}}$, where $m$ is the effective mass of the transmitting electron in the region $\mathrm{A}$ and $\hbar$ is the reduced Planck constant.

As a matter of fact, the most accurate method of computing the tunnelling current through the biased double-barrier structure is based on the exact solution of the Schrödinger wave equation for the piecewise linear potential-energy function $([1-3,12]$ and references cited therein). Usually, the effective-mass approximation with the quadratic momentum-energy relation is supposed to be valid in solving a transmission problem. Second, the electron mean free path is supposed to be longer than the width of the double barrier structure. Further, it is also assumed that the effects of electron-phonon and Coulomb interaction are negligible. By the use of the Airy functions and the transfer matrix technique, the transmission coefficient for the biased double-barrier structure can be computed as a function of the electron longitudinal energy. Modern computers now allow the transmission coefficient for realistic energy-potential functions to be computed with a relative ease. However, an analytical solution of the transmission problem is still of an instructive value. Thus, the need for simple models has not decreased and just the double delta-barrier represents such an archetype of the double barrier structure. This paper presents the derivation of the transmission coefficient in an analytical form and the computation of the tunnelling current through the double delta-barrier.

The organisation of this paper is as follows. In the next section, the Schrödinger wave equation is employed to obtain the wave function for the potential-energy function, which corresponds to the limiting case of the potential barrier shown in Fig. 1. In the third section, the transmission coefficient for the biased double potential delta-barrier is presented. Some attributes of the transmission coefficient are analysed in the fourth section. The tunnelling current is computed in the fifth section. Finally, some concluding remarks are given in the sixth section.

\section{Solution of the Schrödinger equation}

Thus, the one-dimensional stationary Schrödinger wave equation,

$$
\left[-\frac{\hbar^{2} \partial^{2}}{2 m \partial x^{2}}+U(x)-E_{x}\right] \varphi(x)=0,
$$

is solved for the following potential-energy function:

$$
\begin{aligned}
& U(x)=g \delta(x+a)+g \delta(x-a) \\
& \quad-\frac{e V}{2}[\Theta(x+a)-\Theta(x-a)]-e V \Theta(x-a) .
\end{aligned}
$$

Here $x$ represents the longitudinal spatial variable, $E_{x}$ is the longitudinal energy of the conduction electron and $m$ is the electron effective mass. Further, $\delta(x)$ is the Dirac delta-function and $g$ is its strength (a positive value of the strength corresponds to a barrier, while a negative value would correspond to a well), $\Theta(x)$ is the Heaviside step function $(\Theta(x)=0$, if $x<0$; and $\Theta(x)=1$, if $x>0), e$ is the elementary charge, $V$ is the voltage applied to the double delta-barrier, $2 a$ is the width of the double delta-barrier.

Evidently, the wave function $\varphi(x)$ is to be sought in the form of plane waves moving from the left to the right and vice versa [5-14]. Thus,

$$
\begin{aligned}
& \varphi(x)=[1-\Theta(x+a)] \varphi_{\mathrm{I}}(x) \\
& +[\Theta(x+a)-\Theta(x-a)] \varphi_{\mathrm{II}}(x) \\
& +\Theta(x-a) \varphi_{\mathrm{III}}(x) \\
& \quad=[1-\Theta(x+a)]\left(A_{\mathrm{I}} \mathrm{e}^{+\mathrm{i} k_{1} x}+B_{\mathrm{I}} \mathrm{e}^{-\mathrm{i} k_{1} x}\right) \\
& +[\Theta(x+a)-\Theta(x-a)]\left(A_{\mathrm{II}} \mathrm{e}^{+\mathrm{i} k_{2} x}+B_{\mathrm{II}} \mathrm{e}^{-\mathrm{i} k_{2} x}\right) \\
& +\Theta(x-a)\left(A_{\mathrm{III}} \mathrm{e}^{+\mathrm{i} k_{3} x}+B_{\mathrm{III}} \mathrm{e}^{-\mathrm{i} k_{3} x}\right) .
\end{aligned}
$$

The subscript I refers to the spatial region $(-\infty,-a)$ that is on the left-hand side of the biased double delta-barrier, the subscript II refers to spatial region $(-a, a)$ that is in the middle of the biased double delta-barrier and the subscript III refers to the spatial region $(a, \infty)$ that is on the right-hand side of the biased double delta-barrier. The symbols $A_{\mathrm{I}}, B_{\mathrm{I}} ; A_{\mathrm{II}}, B_{\mathrm{II}}$; and $A_{\mathrm{III}}, B_{\mathrm{III}}$ represent the amplitudes of those two plane waves in the respective spatial regions. The three positive wave numbers $k_{1}, k_{2}$ and $k_{3}$ are introduced by the relation $E_{x}=\hbar^{2} k_{1}^{2} / 2 m=\hbar^{2} k_{2}^{2} / 2 m-e V / 2=\hbar^{2} k_{3}^{2} / 2 m-e V$. Evidently, the wave numbers $k_{1}, k_{2}$ and $k_{3}$ represent the longitudinal component of the wave vector in the respective spatial regions. The other two components of the 
wave vectors, $k_{y}$ and $k_{z}$, are parallel to and continuous across the double delta-barrier.

Subjecting the wave function $\varphi(x)$ to the connection formulae at the points $x_{1}=-a$ and $x_{2}=+a$ (vide the Appendix A) one can express it as a sum of three waves: the incident wave, the reflected wave and the transmitted wave [5-12]. Therefore, the wave function on the left-hand side of the biased double delta-barrier can be written as

$$
\begin{gathered}
\varphi_{\mathrm{I}}(x)=A_{\mathrm{I}} \mathrm{e}^{+\mathrm{i} k_{1} x}+A_{\mathrm{I}} r\left(k_{1}, k_{2}, k_{3}\right) \mathrm{e}^{-\mathrm{i} k_{1} x} \\
+B_{\mathrm{III}} t\left(k_{1}, k_{2}, k_{3}\right)\left(\frac{k_{3}}{k_{1}}\right)^{1 / 2} \mathrm{e}^{-\mathrm{i} k_{1} x},
\end{gathered}
$$

and on the right-hand side as

$$
\begin{aligned}
& \varphi_{\mathrm{III}}(x)=B_{\mathrm{III}} \mathrm{e}^{-\mathrm{i} k_{3} x} \\
& -B_{\mathrm{III}} \frac{r^{*}\left(k_{1}, k_{2}, k_{3}\right) t\left(k_{1}, k_{2}, k_{3}\right)}{t^{*}\left(k_{1}, k_{2}, k_{3}\right)} \mathrm{e}^{+i k_{3} x} \\
& +A_{\mathrm{I}} t\left(k_{1}, k_{2}, k_{3}\right)\left(\frac{k_{1}}{k_{3}}\right)^{1 / 2} \mathrm{e}^{+\mathrm{i} k_{3} x} .
\end{aligned}
$$

The quantities $t\left(k_{1}, k_{2}, k_{3}\right)$ and $r\left(k_{1}, k_{2}, k_{3}\right)$ are obtained in Appendix A. Thus,

$$
\begin{aligned}
& \frac{1}{t\left(k_{1}, k_{2}, k_{3}\right)}=\left(\frac{k_{3}}{k_{1}}\right)^{1 / 2} \mathrm{e}^{+\mathrm{i}\left(k_{3}+k_{1}\right) a} \\
& \quad \times\left[\frac{\left(k_{3}-k_{2}+\mathrm{i} \kappa\right)\left(k_{2}-k_{1}-\mathrm{i} \kappa\right)}{4 k_{3} k_{2}} \mathrm{e}^{+2 \mathrm{i} k_{2} a}\right. \\
& \left.\quad+\frac{\left(k_{3}+k_{2}+\mathrm{i} \kappa\right)\left(k_{2}+k_{1}+\mathrm{i} \kappa\right)}{4 k_{3} k_{2}} \mathrm{e}^{-2 \mathrm{i} k_{2} a}\right], \\
& \frac{r\left(k_{1}, k_{2}, k_{3}\right)}{t\left(k_{1}, k_{2}, k_{3}\right)}=-\left(\frac{k_{3}}{k_{1}}\right)^{1 / 2} \mathrm{e}^{+\mathrm{i}\left(k_{3}-k_{1}\right) a} \\
& \quad \times\left[\frac{\left(k_{3}-k_{2}+\mathrm{i} \kappa\right)\left(k_{2}+k_{1}-\mathrm{i} \kappa\right)}{4 k_{3} k_{2}} \mathrm{e}^{+2 \mathrm{i} k_{2} a}\right. \\
& \left.\quad+\frac{\left(k_{3}+k_{2}+\mathrm{i} \kappa\right)\left(k_{2}-k_{1}+\mathrm{i} \kappa\right)}{4 k_{3} k_{2}} \mathrm{e}^{-2 \mathrm{i} k_{2} a}\right],
\end{aligned}
$$

where $\kappa=2 m g / \hbar^{2}$. Evidently, $A_{\text {I }}$ is the amplitude of the plane wave impinging upon the biased double delta-barrier from the left-hand side, $A_{\mathrm{I}} r\left(k_{1}, k_{2}, k_{3}\right)$ gives the amplitude of its reflected wave and $A_{\mathrm{I}} t\left(k_{1}, k_{2}, k_{3}\right) \sqrt{k_{1} / k_{3}}$ the amplitude of its transmitted wave. It is also evident that $B_{\mathrm{III}}$ is the amplitude of the plane wave impinging upon the biased double delta-barrier from the right-hand side, $-B_{\mathrm{III}} r^{*}\left(k_{1}, k_{2}, k_{3}\right) t\left(k_{1}, k_{2}, k_{3}\right) / t^{*}\left(k_{1}, k_{2}, k_{3}\right)$ gives the amplitude of its reflected wave and $B_{\text {III }} t\left(k_{1}, k_{2}, k_{3}\right) \sqrt{k_{3} / k_{1}}$ the amplitude of its transmitted wave. Obviously (vide also Appendix B), the quantities $t\left(k_{1}, k_{2}, k_{3}\right)$ and $r\left(k_{1}, k_{2}, k_{3}\right)$, respectively, give the transmission amplitude and the reflection amplitude. Actually, $r\left(k_{1}, k_{2}, k_{3}\right)$ is the reflection amplitude from the left and $-r^{*}\left(k_{1}, k_{2}, k_{3}\right) t\left(k_{1}, k_{2}, k_{3}\right) / t^{*}\left(k_{1}, k_{2}, k_{3}\right)$ is the reflection amplitude from the right, they differ only in a phase. The transmission coefficient is defined as $T\left(k_{1}, k_{2}, k_{3}\right)=t^{*}\left(k_{1}, k_{2}, k_{3}\right) t\left(k_{1}, k_{2}, k_{3}\right)$. Evidently, the reflection coefficient is given by $R\left(k_{1}, k_{2}, k_{3}\right)=$ $r^{*}\left(k_{1}, k_{2}, k_{3}\right) r\left(k_{1}, k_{2}, k_{3}\right)=1-T\left(k_{1}, k_{2}, k_{3}\right)$.

\section{Transmission coefficient}

After straightforward algebra, one can derive the transmission coefficient $T\left(k_{1}, k_{2}, k_{3}\right)$ for the biased double delta-barrier in an analytical form. It is to be obtained from the following expression:

$$
\begin{aligned}
& \frac{4 k_{3} k_{2}^{2} k_{1}}{T\left(k_{1}, k_{2}, k_{3}\right)}=4 k_{3} k_{2}^{2} k_{1} \\
& \quad+\left(k_{3}-k_{1}\right)^{2}\left[k_{2} \cos \left(2 k_{2} a\right)+\kappa \sin \left(2 k_{2} a\right)\right]^{2} \\
& \quad+\left[2 \kappa k_{2} \cos \left(2 k_{2} a\right)+\left(\kappa^{2}-k_{2}^{2}+k_{3} k_{1}\right) \sin \left(2 k_{2} a\right)\right]^{2} .
\end{aligned}
$$

If $a=0$, one gets the transmission coefficient for the biased single delta-barrier of the twofold strength [13]:

$$
T_{a=0}\left(k_{1}, k_{2}, k_{3}\right)=\frac{4 k_{3} k_{1}}{\left(k_{3}+k_{1}\right)^{2}+(2 \kappa)^{2}} .
$$

Putting $k_{3}=k_{1}=\sqrt{2 m E_{x}} / \hbar$ in $T\left(k_{1}, k_{2}, k_{1}\right)$ one easily obtains the transmission coefficient for the double delta-barrier with a rectangular well in between [11]; the well has the depth $\mathrm{eV} / 2$ and the width $2 a$,

$$
\begin{aligned}
& T\left(k_{1}, k_{2}, k_{1}\right)=\left(4 k_{2}^{2} k_{1}^{2}\right) /\left\{4 k_{2}^{2} k_{1}^{2}\right. \\
& \left.\quad+\left[2 \kappa k_{2} \cos \left(2 k_{2} a\right)+\left(\kappa^{2}-k_{2}^{2}+k_{1}^{2}\right) \sin \left(2 k_{2} a\right)\right]^{2}\right\} .
\end{aligned}
$$

If there is no external voltage applied to the double delta-barrier, i.e. if $V=0$, then $k_{3}=k_{2}=$ $k_{1}=\sqrt{2 m E_{x}} / \hbar=k$ and the transmission coefficient $T\left(k_{1}, k_{2}, k_{3}\right)$ is reduced to the transmission coefficient for the unbiased double delta-barrier [8-11],

$$
T(k, k, k)=\frac{4 k^{4}}{4 k^{4}+\kappa^{2}\left[2 k \cos \left(2 k_{2} a\right)+\kappa \sin \left(2 k_{2} a\right)\right]^{2}} .
$$

If $\kappa=0$, one gets the transmission coefficient for the two-step rectangular well, the first well has the depth $e V / 2$ and the width $2 a$, the second well has the depth $e V$ and extends to infinity,

$$
\begin{aligned}
& T_{\kappa=0}\left(k_{1}, k_{2}, k_{3}\right)=\left(4 k_{3} k_{2}^{2} k_{1}\right) /\left\{4 k_{3} k_{2}^{2} k_{1}\right. \\
& \left.+\left(k_{3}-k_{1}\right)^{2} k_{2}^{2} \cos ^{2}\left(2 k_{2} a\right)+\left(k_{2}^{2}-k_{3} k_{1}\right)^{2} \sin ^{2}\left(2 k_{2} a\right)\right\} .
\end{aligned}
$$

If $\kappa=0$ as well as $a=0$, one gets the transmission coefficient for the one-step rectangular well $[5,6,13]$.

\section{Attributes of the transmission coefficient}

In Fig. 2, the transmission coefficient $T\left(k_{1}, k_{2}, k_{3}\right)$ as a function of the longitudinal energy $E_{x}$ of the transmitting electron is presented for four different applied voltages $V$. The effective mass $m$ of the transmitting electron is supposed to be $0.067 m_{0}$, where $m_{0}$ is the free electron mass; the width $2 a$ of the biased double delta-barrier is taken as $5.0 \times 10^{-9} \mathrm{~m}$, and its strength $g$ as $\left(2.0 \times 10^{-9} \mathrm{~m}\right)(0.50 \mathrm{eV})$. These numerical values are also used in the other calculations. It is seen that the transition coefficient $T\left(k_{1}, k_{2}, k_{3}\right)$ exhibits relative maxima and relative minima. Outside the strong lower-energy peaks at small applied voltages, the transmission coefficient is clearly a smooth function of the longitudinal energy of the transmitting electron. 


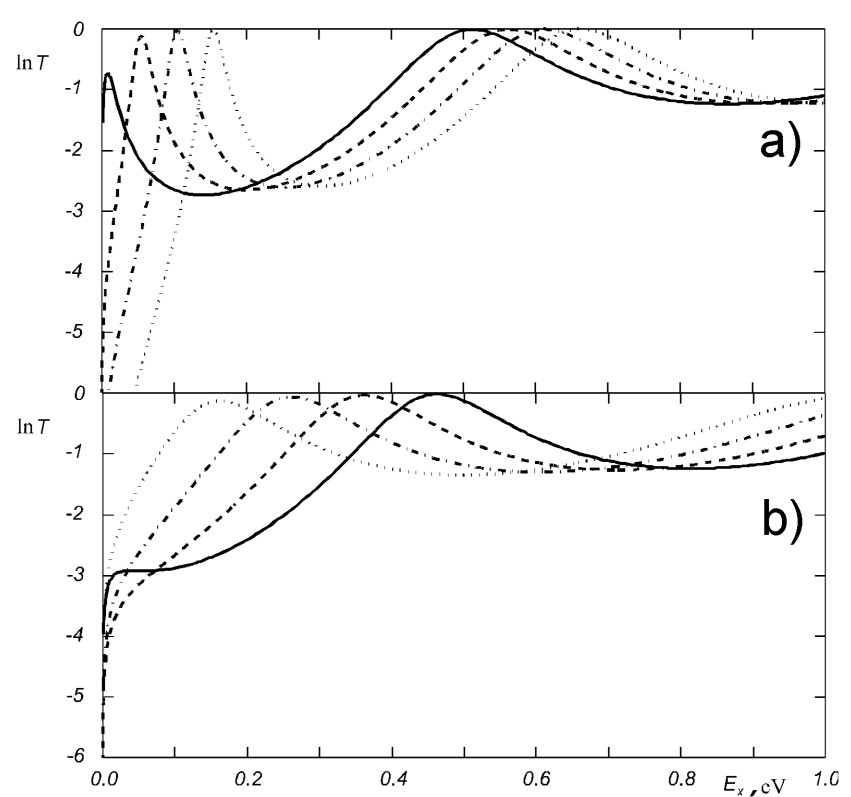

Fig. 2. (a) and (b) Natural logarithm of the transmission coefficient for the biased double delta-barrier at various applied voltages $V$ versus the longitudinal energy of the transmitting electron, the barrier width is $5.0 \times 10^{-9} \mathrm{~m}$. In (a), the dotted curve is at $V=0.00 \mathrm{~V}$, the dash-dotted curve at $V=0.10 \mathrm{~V}$, the dashed curve at $V=0.20 \mathrm{~V}$, the full curve at $V=0.30 \mathrm{~V}$; in (b), the full curve is at $V=0.40 \mathrm{~V}$, the dashed curve at $V=0.60 \mathrm{~V}$, the dash-dotted curve at $V=0.80 \mathrm{~V}$, the dotted curve at $V=1.00 \mathrm{~V}$.

Evidently, two independent resonance conditions must be satisfied simultaneously so that the transmission coefficient $T\left(k_{1}, k_{2}, k_{3}\right)$ could be equal to unity, i.e. a transmission through the biased double delta-barrier could be ideal. One is the maximum condition for the peak value, $2 \kappa k_{2} \cos \left(2 k_{2} a\right)+\left(\kappa^{2}-k_{2}^{2}+k_{3} k_{1}\right) \sin \left(2 k_{2} a\right)=0$, and the other is the phase-difference condition, $k_{2} \cos \left(2 k_{2} a\right)+$ $\kappa \sin \left(2 k_{2} a\right)=0$. However, these two resonance conditions cannot be satisfied simultaneously. Thus, a transmission through the biased double delta-barrier never becomes ideal (in the limit $E_{x} \rightarrow \infty$, the transmission coefficient $T\left(k_{1}, k_{2}, k_{3}\right)$ goes to unity).

To find local extremes in the transmission coefficient $T\left(k_{1}, k_{2}, k_{3}\right)$, one has to find the first derivative of $T\left(k_{1}, k_{2}, k_{3}\right)$ with respect to $E_{x}$ and equate it to zero. It is valid,

$$
\begin{aligned}
& \frac{\mathrm{d} T\left(k_{1}, k_{2}, k_{3}\right)}{\mathrm{d} E_{x}} \cong \frac{a T^{2}\left(k_{1}, k_{2}, k_{3}\right)}{k_{3} k_{2}^{2} k_{1}} \frac{\mathrm{d} k_{2}}{\mathrm{~d} E_{x}} \\
& \quad \times\left[2 \kappa k_{2} \cos \left(2 k_{2} a\right)+\left(\kappa^{2}-k_{2}^{2}+k_{3} k_{1}\right) \sin \left(2 k_{2} a\right)\right] \\
& \quad \times\left[2 \kappa k_{2} \sin \left(2 k_{2} a\right)-\left(\kappa^{2}-k_{2}^{2}+k_{3} k_{1}\right) \cos \left(2 k_{2} a\right)\right] .
\end{aligned}
$$

This approximation for first derivative is justifiable only when the double delta-barrier is wide enough and is under the application of a small external voltage, i.e. when $1 \ll a \sqrt{2 m E_{x}} / \hbar$ and $e V \ll E_{x}$.
Thus, the incomplete resonant energy $E_{x, \max }$ i.e. the energy, at which the transmission coefficient $T\left(k_{1}, k_{2}, k_{3}\right)$ has a relative maximum, approximately obeys the maximum condition for the peak value, $2 \kappa k_{2} \cos \left(2 k_{2} a\right)+\left(\kappa^{2}-\right.$ $\left.k_{2}^{2}+k_{3} k_{1}\right) \sin \left(2 k_{2} a\right)=0$. When this maximum condition is satisfied, the transmission coefficient $T\left(k_{1}, k_{2}, k_{3}\right)$ takes the form

$$
\begin{aligned}
& T_{\max }\left(k_{1}, k_{2}, k_{3}\right) \\
& =\frac{4 k_{3} k_{1}}{4 k_{3} k_{1}+\frac{\left(k_{3}-k_{1}\right)^{2}\left(\kappa^{2}+k_{2}^{2}-k_{3} k_{1}\right)^{2}}{4 \kappa^{2} k_{2}^{2}+\left(\kappa^{2}-k_{2}^{2}+k_{3} k_{1}\right)^{2}}} .
\end{aligned}
$$

It is easy to obtain that $T_{a=0}\left(k_{1}, k_{2}, k_{3}\right)<$ $T_{\max }\left(k_{1}, k_{2}, k_{3}\right)$ as far as $\kappa \neq 0$. If $\kappa=0$ then $T_{a=0}\left(k_{1}, k_{2}, k_{3}\right)=T_{\max }\left(k_{1}, k_{2}, k_{3}\right)$. In the range of small applied voltages, when $e V \ll E_{x}$, then $k_{3} \cong k_{1}$ is valid, and therefore $T_{\max }\left(k_{1}, k_{2}, k_{3}\right) \cong 1$.

It should be recalled that the ideal transmission through the double barrier structure (the transmission without a reflection) is a result of the constructive interference between the waves just transmitting through the first barrier and those being reflected off the second one. The stronger the barriers are, the smaller the intensity of the transmitted waves is and the more pronounced the interference becomes. In the case of the transmissions through the biased double delta-barrier, there also exists some constructive interference between the waves just transmitting through the first delta-barrier and those being reflected off the second one. Unlike the transmissions through the unbiased double delta-barrier [9-11], the constructive interference in the biased double delta-barrier is not strong enough to cause the ideal transmission. Thus, the applied voltage reduces the constructive interference between the waves in the double delta-barrier. The constructive interference between the waves is also reduced in the asymmetrical double delta-barrier [14]. Generally, the asymmetry reduces the constructive interference [1]. From Fig. 2 it is seen that the higher the applied voltage, the smaller is the lower incomplete resonant energy. The lower-energy peak, however, disappears at an applied voltage of about $0.4 \mathrm{~V}$.

Evidently, the energy $E_{x, \text { min }}$, at which the transmission coefficient $T\left(k_{1}, k_{2}, k_{3}\right)$ has a relative minimum, should approximately obey the minimum condition for the valley value, $2 \kappa k_{2} \sin \left(2 k_{2} a\right)-\left(\kappa^{2}-k_{2}^{2}+\right.$ $\left.k_{3} k_{1}\right) \cos \left(2 k_{2} a\right)=0$. If this minimum condition is satisfied, the transmission coefficient $T\left(k_{1}, k_{2}, k_{3}\right)$ can be arranged into the next form

$$
\begin{aligned}
& T_{\min }\left(k_{1}, k_{2}, k_{3}\right) \\
& =\frac{4 k_{3} k_{2}^{2} k_{1}}{\left(\kappa^{2}+k_{2}^{2}+k_{3} k_{1}\right)^{2}+\frac{\left(\kappa^{2}+k_{2}^{2}+k_{3} k_{1}\right)^{2}\left(k_{3}-k_{1}\right)^{2} \kappa^{2}}{4 \kappa^{2} k_{2}^{2}+\left(\kappa^{2}-k_{2}^{2}+k_{3} k_{1}\right)^{2}}} .
\end{aligned}
$$

There also exists some destructive interference between the waves just transmitting through the first delta-barrier and those being reflected off the second one. Therefore, one expects that $T_{\min }\left(k_{1}, k_{2}, k_{3}\right)<T_{a=0}\left(k_{1}, k_{2}, k_{3}\right)$. This inequality between the transmission coefficients $T_{\min }\left(k_{1}, k_{2}, k_{3}\right)$ and $T_{a=0}\left(k_{1}, k_{2}, k_{3}\right)$ is satisfied as far as 


$$
\begin{aligned}
& \left(k_{3}-k_{1}\right)^{2} \kappa^{4} k_{3} k_{1}+\frac{\left(k_{3}-k_{1}\right)^{6}\left(2 \kappa^{2}+k_{2}^{2}+k_{3} k_{1}\right)}{8} \\
& <\kappa^{8}+2 \kappa^{6}\left(k_{2}^{2}+k_{3} k_{1}\right) .
\end{aligned}
$$

In the range of small applied voltages, when $k_{3} \cong k_{1}$, the previous inequality is certainly satisfied. It is also satisfied if the transmissions occur through the double delta-barrier that is enough strong, i.e. if $k \ll \kappa$. In such cases, it is to be expected that the minimum condition of the transmission coefficient $T\left(k_{1}, k_{2}, k_{3}\right)$ is properly expressed by the minimum condition for the valley value, and the transmission coefficient $T_{\min }\left(k_{1}, k_{2}, k_{3}\right)$ gives a proper value of the transmission coefficient $T\left(k_{1}, k_{2}, k_{3}\right)$ in its minimum.

In Fig. 3 the transmission coefficients $T\left(k_{1}, k_{2}, k_{3}\right)$, $T_{\min }\left(k_{1}, k_{2}, k_{3}\right), T_{\max }\left(k_{1}, k_{2}, k_{3}\right)$, and $T_{a=0}\left(k_{1}, k_{2}, k_{3}\right)$ are drawn as a function of the longitudinal energy $E_{x}$ at the applied voltage of $0.30 \mathrm{~V}$. In Fig. 4 they are drawn as a function of the applied voltage $V$; the longitudinal energy $E_{x}$ of the transmitting electron is taken as $0.0025 \mathrm{eV}$. In both the figures, the full curve depicts the transmission coefficient $T\left(k_{1}, k_{2}, k_{3}\right)$, the dashed curve shows the transmission coefficient $T_{a=0}\left(k_{1}, k_{2}, k_{3}\right)$ and the dotted curves show the transmission coefficients $T_{\max }\left(k_{1}, k_{2}, k_{3}\right)$ and $T_{\min }\left(k_{1}, k_{2}, k_{3}\right)$. These curves demonstrate the validity of the inequality $T_{\min }\left(k_{1}, k_{2}, k_{3}\right)<T_{a=0}\left(k_{1}, k_{2}, k_{3}\right)<$ $T_{\max }\left(k_{1}, k_{2}, k_{3}\right)$. It is also seen that the transmission coefficients $T_{\min }\left(k_{1}, k_{2}, k_{3}\right)$ and $T_{\max }\left(k_{1}, k_{2}, k_{3}\right)$, respectively, trace minima and maxima of the transmission coefficient $T\left(k_{1}, k_{2}, k_{3}\right)$ rather well, at least at low applied voltages.

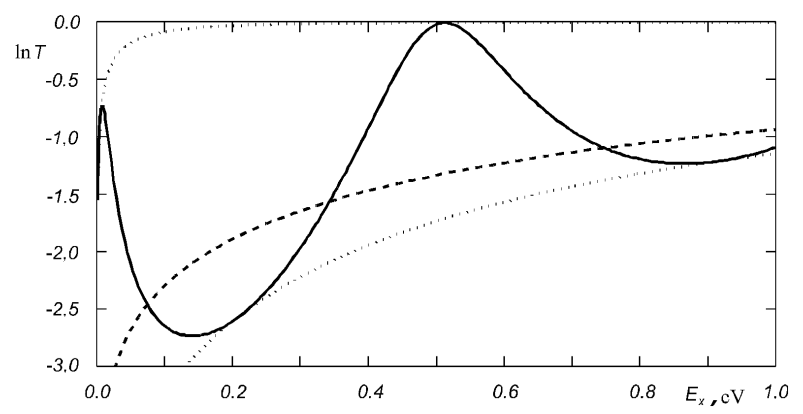

Fig. 3. Natural logarithm of the transmission coefficient at the applied voltage of $0.30 \mathrm{~V}$ versus the longitudinal energy of the transmitting electron, the barrier width is $5.0 \times 10^{-9} \mathrm{~m}$. The full curve is the transmission coefficient for the biased double delta-barrier, the dashed curve is the transmission coefficient for the biased single delta-barrier of the twofold strength, and the dotted curves are the transmission coefficients giving the maximum and minimum value of the transmission probability.

In Fig. 5 the transmission coefficient $T\left(k_{1}, k_{2}, k_{3}\right)$ as a function of the applied voltage $V$ is presented for three different widths $2 a$ of the biased double delta-barrier; the longitudinal energy $E_{x}$ of the transmitting electron is again taken as $0.0025 \mathrm{eV}$. It is seen that the barrier width $2 a$ strongly influences both the voltage $V_{\max }$ and the voltage $V_{\min }$, at which the local maximum and lo-

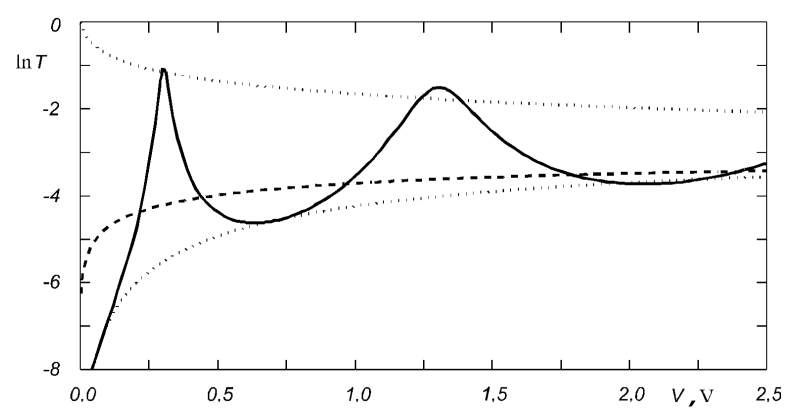

Fig. 4. Natural logarithm of the transmission coefficient at the longitudinal energy of $0.0025 \mathrm{eV}$ versus the applied voltage, the barrier width is $5.0 \times 10^{-9} \mathrm{~m}$. The full curve is the transmission coefficient for the biased double delta-barrier, the dashed curve is the transmission coefficient for the biased single delta-barrier of the twofold strength, and the dotted curves are the transmission coefficients giving the maximum and minimum value of the transmission probability.

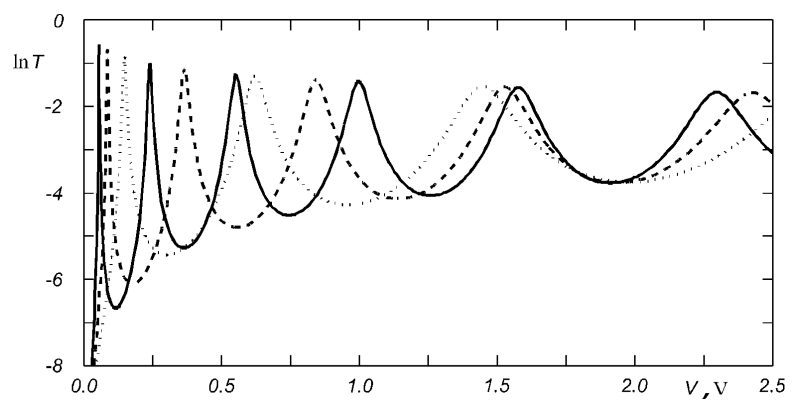

Fig. 5. Natural logarithm of the transmission coefficient at the longitudinal energy of $0.0025 \mathrm{eV}$ versus the applied voltage for three different values of the barrier width $2 a$; the full curve is for $2 a=12.5 \times 10^{-9} \mathrm{~m}$, the dashed curve for $2 a=10.0 \times 10^{-9} \mathrm{~m}$, the dotted curve for $2 a=7.5 \times 10^{-9} \mathrm{~m}$.

cal minimum occur, respectively. The graphs show that they are shifted towards smaller values in a wider barrier. As it is expected, the number of maxima and minima is increased in the wider double delta-barrier.

All the attributes of the transmission coefficient for the biased double delta-barrier are expected to manifest themselves in the current-voltage characteristics of the biased double delta-barrier.

\section{Tunnelling current}

Generally (vide e.g. [1, 2]), the current density through a biased barrier structure may be computed as

$$
\begin{aligned}
& j(V)=\frac{4 \pi m e}{(2 \pi \hbar)^{3}} \int_{0}^{\infty} \mathrm{d} E \int_{0}^{E}[f(E)-f(E+e V)] \\
& \quad \times T\left(E_{x}, V\right) \mathrm{d} E_{x} .
\end{aligned}
$$

Here $E$ is the energy of the transmitting electron measured from the bottom of the conduction band of the unbiased region of the barrier structure. Further, $f(E)=$ 
$\left[1+\exp \left(\left(E-E_{\mathrm{F}}\right) / k_{\mathrm{B}} \theta\right)\right]^{-1}$ is the Fermi-Dirac distribution function at the absolute temperature $\theta$ with $E_{\mathrm{F}}$ being the Fermi energy and $k_{\mathrm{B}}$ is the Boltzmann constant; $V$ is the voltage applied to the structure, $T\left(E_{x}, V\right) \equiv$ $T\left(k_{1}, k_{2}, k_{3}\right)$ is the transmission coefficient as a function of the longitudinal energy $E_{x}$ of the transmitting electron at the applied voltage $V$. In the limit $\theta \rightarrow 0$, the above expression for the current density becomes

$$
\begin{aligned}
& j(V)=\frac{4 \pi m e}{(2 \pi \hbar)^{3}}\left[\int_{0}^{E_{\mathrm{F}}}\left(E_{\mathrm{F}}-E_{x}\right) T\left(E_{x}, V\right) \mathrm{d} E_{x}\right. \\
& -\Theta\left(E_{\mathrm{F}}-e V\right) \int_{0}^{E_{\mathrm{F}}-e V}\left(E_{\mathrm{F}}-e V-E_{x}\right) \\
& \left.\quad \times T\left(E_{x}, V\right) \mathrm{d} E_{x}\right] .
\end{aligned}
$$

Figure 6 shows the calculated current density through the biased double delta-barrier at zero absolute temperature as a function of the applied voltage (the numerical value of $j_{0}$ is to be calculated from the expression $\left.j_{0}=4 \pi m e E_{\mathrm{F}}^{2} /(2 \pi \hbar)^{3}\right)$. In the calculation, the Fermi energy $E_{\mathrm{F}}$ is assumed to have a low value of $0.0050 \mathrm{eV}$. The current density in Fig. 6 shows almost the identical oscillatory behaviour as the transmission coefficient in Fig. 4 when the applied voltage is increased. This oscillatory behaviour is also demonstrated in Fig. 7 where the calculated current density at zero absolute temperature is drawn as a function of the applied voltage for three different widths $2 a$ of the biased double delta-barrier. It is seen that the graphs in Fig. 7 are very similar to those in Fig. 5.

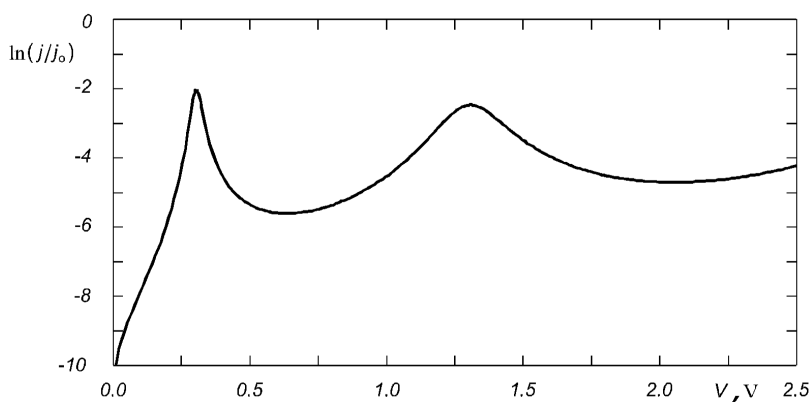

Fig. 6. Natural logarithm of the current density through the biased double delta-barrier at zero absolute temperature versus the applied voltage, the barrier width is $5.0 \times 10^{-9} \mathrm{~m}$.

For a very low value of the Fermi energy, when the transmission coefficient $T\left(E_{x}, V\right)$ at a particular voltage $V$ smoothly varies with the longitudinal energy $E_{x}$ on the interval from zero to the Fermi energy, one has $j(V) \cong 2 \pi m e E_{\mathrm{F}}^{2} T\left(E_{\mathrm{F}} / 2, V\right) /(2 \pi \hbar)^{3}=j_{0} T\left(E_{\mathrm{F}} / 2, V\right) / 2$. That is the reason why the graph of $j(V)$ is very similar to the graph of $T\left(E_{\mathrm{F}} / 2, V\right)$. Thus, when the Fermi energy is very low, it can be said that a maximum in current density, which is accompanied by a region of negative differential conductance, appears at the applied voltage $V_{\max }$ at which the incomplete resonant energy $E_{x, \max }$ of the biased double delta-barrier matches the Fermi energy. It

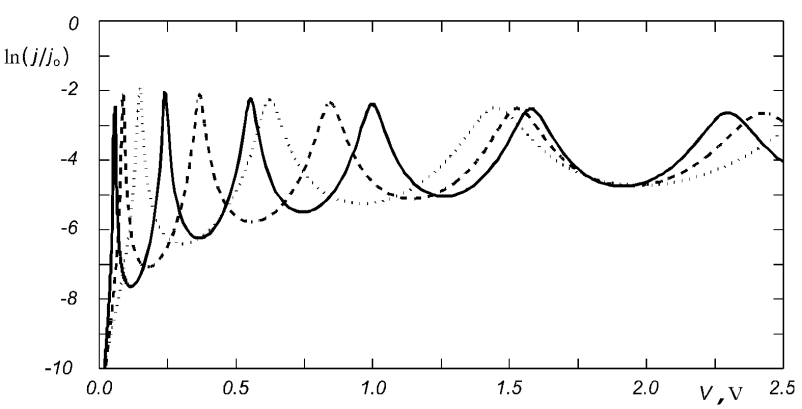

Fig. 7. Natural logarithm of the current density through the biased double delta-barrier at zero absolute temperature versus the applied voltage for three different values of the barrier width $2 a$; the full curve is for $2 a=12.5 \times 10^{-9} \mathrm{~m}$, the dashed curve for $2 a=$ $10.0 \times 10^{-9} \mathrm{~m}$, the dotted curve for $2 a=7.5 \times 10^{-9} \mathrm{~m}$.

is also evident that a minimum in current density appears at the applied voltage $V_{\min }$ when the energy $E_{x, \min }$, at which the local minimum in the transmission coefficient for the biased double delta-barrier occurs, matches the Fermi energy.

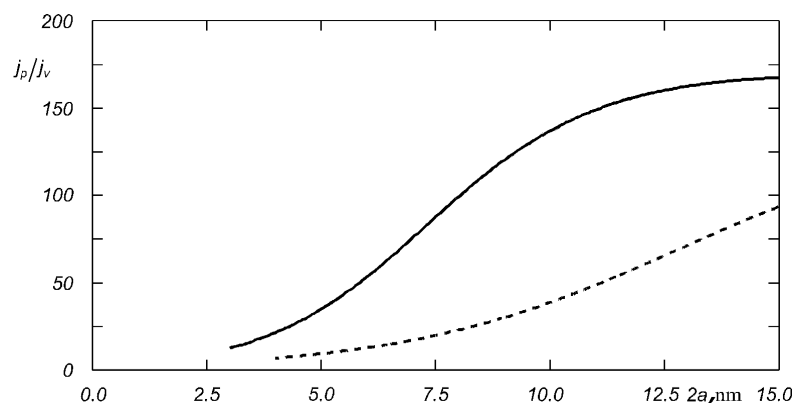

Fig. 8. The peak-to-valley ratio in the current-voltage characteristics versus the barrier width; the full curve is for the first peak and the first valley, the dashed curve for the second peak and the second valley.

The graphs in Fig. 7 also suggest that the ratio of the peak value to the valley value in the currentvoltage characteristics can be modulated with the barrier width. For a very low value of the Fermi energy, the peak-to-valley ratio is approximately given by $j_{\mathrm{p}} / j_{\mathrm{v}} \cong T\left(E_{\mathrm{F}} / 2, V_{\max }\right) / T\left(E_{\mathrm{F}} / 2, V_{\min }\right)$. In Fig. 8 the calculated peak-to-valley ratio is presented as a function of the width $2 a$ of the double delta-barrier. The full curve shows the peak-to-valley ratio for the first peak and the first valley, the dashed curve for the second peak and the second valley. Both the calculated curves are very smooth. Thus, the barrier width can easily be chosen to give a particular value of the peak-to-valley ratio.

\section{Concluding remarks}

We presented the solution to the transmission problem in the biased double delta-barrier that represents a simple 
model of the biased double barrier structure. The exact formula of the transmission coefficient has been employed for the numerical calculation of the current density. The numerical results show that the current density exhibits the same oscillatory behaviour as the transmission coefficient. Thus, the negative differential resistance in the current-voltage characteristics appears at a voltage that corresponds to a transmission-coefficient peak. The numerical results also show that a particular value of the peak-to-valley ratio in the current-voltage characteristics can be obtained with the modulation of the barrier width. The numerical values used throughout the calculation, the effective mass $m$ of the transmitting particle and the Fermi energy $E_{\mathrm{F}}$, correspond to GaAs, while the strength $g$ of the delta-barriers to $\mathrm{Al}_{1-x} \mathrm{Ga}_{x} \mathrm{As}$, vide e.g. [1-3]. The calculation can easily be extended to other similar sandwich structures.

The great advantage of this simple model is that the transmission coefficient can be calculated analytically. However, the possible variations in the width and in the height of the biased double barrier structure are taken into account only in a very simple way and the effective-mass variation in the double barrier structure is fully lost in this approach. Still, similar numerical results were obtained for more realistic model of the biased double barrier structure [1-3].

\section{Appendix A}

Two connection formulae are to be obtained from the continuity condition for the wave function $\varphi(x)$ at the points $x_{1}=-a$ and $x_{2}=+a$. It is valid, $\varphi(-a+0)=$ $\varphi(-a-0)$ and $\varphi(a+0)=\varphi(a-0)$. Thus,

$$
\begin{aligned}
& A_{\mathrm{I}} \mathrm{e}^{-\mathrm{i} k_{1} a}+B_{\mathrm{I}} \mathrm{e}^{+\mathrm{i} k_{1} a}=A_{\text {II }} \mathrm{e}^{-\mathrm{i} k_{2} a}+B_{\mathrm{II}} \mathrm{e}^{+\mathrm{i} k_{2} a}, \\
& A_{\mathrm{II}} \mathrm{e}^{+\mathrm{i} k_{2} a}+B_{\text {II }} \mathrm{e}^{-\mathrm{i} k_{2} a}=A_{\text {III }} \mathrm{e}^{+\mathrm{i} k_{3} a}+B_{\text {III }} \mathrm{e}^{-\mathrm{i} k_{3} a} .
\end{aligned}
$$

A formal integration of the Schrödinger equation around the points $x_{1}=-a$ and $x_{2}=+a$ leads to the relations, which express the discontinuity of the first derivative of the wave function at those two points; vide e.g. $[8,9]$. It is valid, $\varphi^{\prime}(-a+0)-\varphi^{\prime}(-a-0)=2 m g \varphi(-a) / \hbar^{2}$ and $\varphi^{\prime}(a+0)-\varphi^{\prime}(a-0)=2 m g \varphi(a) / \hbar^{2}$, where $\varphi^{\prime}(x)=$ $\mathrm{d} \varphi(x) / \mathrm{d} x$. These two discontinuity relations yield two other connection formulae. Thus,

$$
\begin{gathered}
\mathrm{i} k_{2}\left(A_{\text {II }} \mathrm{e}^{-\mathrm{i} k_{2} a}-B_{\text {II }} \mathrm{e}^{+\mathrm{i} k_{2} a}\right) \\
-\mathrm{i} k_{1}\left(A_{\mathrm{I}} \mathrm{e}^{-\mathrm{i} k_{1} a}-B_{\mathrm{I}} \mathrm{e}^{+\mathrm{i} k_{1} a}\right) \\
=\kappa\left(A_{\mathrm{I}} \mathrm{e}^{-\mathrm{i} k_{1} a}+B_{\mathrm{I}} \mathrm{e}^{+\mathrm{i} k_{1} a}\right), \\
\mathrm{i} k_{3}\left(A_{\text {III }} \mathrm{e}^{+\mathrm{i} k_{3} a}-B_{\text {III }} \mathrm{e}^{-\mathrm{i} k_{3} a}\right) \\
-\mathrm{i} k_{2}\left(A_{\mathrm{II}} \mathrm{e}^{+\mathrm{i} k_{2} a}-B_{\mathrm{II}} \mathrm{e}^{-\mathrm{i} k_{2} a}\right) \\
=\kappa\left(A_{\mathrm{II}} \mathrm{e}^{+\mathrm{i} k_{2} a}+B_{\mathrm{II}} \mathrm{e}^{-\mathrm{i} k_{2} a}\right),
\end{gathered}
$$

where $\kappa=2 m g / \hbar^{2}$.

It is appropriate to use the transfer-matrix method $[1,12]$. Therefore, the connection formulae are to be rewritten in the form of matrices

$$
\begin{aligned}
& {\left[\begin{array}{l}
A_{\mathrm{II}} \\
B_{\mathrm{II}}
\end{array}\right]=M(\mathrm{II}, \mathrm{I})\left[\begin{array}{l}
A_{\mathrm{I}} \\
B_{\mathrm{I}}
\end{array}\right],} \\
& {\left[\begin{array}{l}
A_{\mathrm{III}} \\
B_{\mathrm{III}}
\end{array}\right]=M(\mathrm{III}, \mathrm{II})\left[\begin{array}{l}
A_{\mathrm{II}} \\
B_{\mathrm{II}}
\end{array}\right] .}
\end{aligned}
$$

The elements of the transfer matrices $M(\mathrm{II}, \mathrm{I})$ and $M(\mathrm{III}, \mathrm{II})$ are given by

$$
\begin{aligned}
& M_{11}(\mathrm{II}, \mathrm{I})=M_{22}^{*}(\mathrm{II}, \mathrm{I})=\frac{k_{2}+k_{1}-\mathrm{i} \kappa}{2 k_{2}} \mathrm{e}^{+\mathrm{i}\left(k_{2}-k_{1}\right) a}, \\
& M_{12}(\mathrm{II}, \mathrm{I})=M_{21}^{*}(\mathrm{II}, \mathrm{I})=\frac{k_{2}-k_{1}-\mathrm{i} \kappa}{2 k_{2}} \mathrm{e}^{+\mathrm{i}\left(k_{2}+k_{1}\right) a}, \\
& M_{11}(\mathrm{III}, \mathrm{II})=M_{22}^{*}(\mathrm{III}, \mathrm{II})=\frac{k_{3}+k_{2} \mathrm{i} \kappa}{2 k_{3}} \mathrm{e}^{-\mathrm{i}\left(k_{3}-k_{2}\right) a}, \\
& M_{12}(\mathrm{III}, \mathrm{II})=M_{21}^{*}(\mathrm{III}, \mathrm{II})=\frac{k_{3}-k_{2}-\mathrm{i} \kappa}{2 k_{3}} \mathrm{e}^{-\mathrm{i}\left(k_{3}+k_{2}\right) a} .
\end{aligned}
$$

It is easy to show that $\operatorname{det} M(\mathrm{II}, \mathrm{I})=k_{1} / k_{2}$ and $\operatorname{det} M(\mathrm{III}, \mathrm{II})=k_{2} / k_{3}$.

The two consecutive transfers are to be joined into one,

$$
\left[\begin{array}{l}
A_{\mathrm{III}} \\
B_{\mathrm{III}}
\end{array}\right]=M(\mathrm{III}, \mathrm{II}) M(\mathrm{II}, \mathrm{I})\left[\begin{array}{l}
A_{\mathrm{I}} \\
B_{\mathrm{I}}
\end{array}\right]=M(\mathrm{III}, \mathrm{I})\left[\begin{array}{l}
A_{\mathrm{I}} \\
B_{\mathrm{I}}
\end{array}\right],
$$

where

$$
\begin{aligned}
& M_{11}(\mathrm{III}, \mathrm{I})=M_{22}^{*}(\mathrm{III}, \mathrm{I}) \\
& =\frac{\left(k_{3}+k_{2}-\mathrm{i} \kappa\right)\left(k_{2}+k_{1}-\mathrm{i} \kappa\right)}{4 k_{3} k_{2}} \mathrm{e}^{-\mathrm{i}\left(k_{1}-2 k_{2}+k_{3}\right) a} \\
& +\frac{\left(k_{3}-k_{2}-\mathrm{i} \kappa\right)\left(k_{2}-k_{1}+\mathrm{i} \kappa\right)}{4 k_{3} k_{2}} \mathrm{e}^{-\mathrm{i}\left(k_{1}+2 k_{2}+k_{3}\right) a} \\
& =\left(\frac{k_{1}}{k_{3}}\right)^{1 / 2} \frac{1}{t^{*}\left(k_{1}, k_{2}, k_{3}\right)}, \\
& M_{12}(\mathrm{III}, \mathrm{I})=M_{21}^{*}(\mathrm{III}, \mathrm{I}) \\
& =\frac{\left(k_{3}+k_{2}-\mathrm{i} \kappa\right)\left(k_{2}-k_{1}-\mathrm{i} \kappa\right)}{4 k_{3} k_{2}} \mathrm{e}^{+\mathrm{i}\left(k_{1}+2 k_{2}-k_{3}\right) a} \\
& +\frac{\left(k_{3}-k_{2}-\mathrm{i} \kappa\right)\left(k_{2}+k_{1}+\mathrm{i} \kappa\right)}{4 k_{3} k_{2}} \mathrm{e}^{+\mathrm{i}\left(k_{1}-2 k_{2}-k_{3}\right) a} \\
& =-\left(\frac{k_{1}}{k_{3}}\right)^{1 / 2} \frac{r^{*}\left(k_{1}, k_{2}, k_{3}\right)}{t^{*}\left(k_{1}, k_{2}, k_{3}\right)} .
\end{aligned}
$$

It is easy to obtain that

$$
\begin{aligned}
& \operatorname{det} M(\mathrm{III}, \mathrm{I})=\operatorname{det} M(\mathrm{III}, \mathrm{II}) \operatorname{det} M(\mathrm{II}, \mathrm{I})=\frac{k_{1}}{k_{3}} \\
& =\frac{k_{1}}{k_{3}} \frac{1-r\left(k_{1}, k_{2}, k_{3}\right) r^{*}\left(k_{1}, k_{2}, k_{3}\right)}{t\left(k_{1}, k_{2}, k_{3}\right) t^{*}\left(k_{1}, k_{2}, k_{3}\right)} .
\end{aligned}
$$

Evidently, $r\left(k_{1}, k_{2}, k_{3}\right) r^{*}\left(k_{1}, k_{2}, k_{3}\right)=1-t\left(k_{1}, k_{2}, k_{3}\right)$ $t^{*}\left(k_{1}, k_{2}, k_{3}\right)$ is valid. The new-introduced quantities $t\left(k_{1}, k_{2}, k_{3}\right)$ and $r\left(k_{1}, k_{2}, k_{3}\right)$ enable one to express the relation between the amplitudes in the first and third spatial region in the very simple form,

$$
B_{\mathrm{I}}=A_{\mathrm{I}} r\left(k_{1}, k_{2}, k_{3}\right)+B_{\mathrm{III}} t\left(k_{1}, k_{2}, k_{3}\right)\left(\frac{k_{3}}{k_{1}}\right)^{1 / 2},
$$




$$
\begin{gathered}
A_{\mathrm{III}}=A_{\mathrm{I}} t\left(k_{1}, k_{2}, k_{3}\right)\left(\frac{k_{1}}{k_{3}}\right)^{1 / 2} \\
-B_{\mathrm{III}} \frac{r^{*}\left(k_{1}, k_{2}, k_{3}\right) t\left(k_{1}, k_{2}, k_{3}\right)}{t^{*}\left(k_{1}, k_{2}, k_{3}\right)} .
\end{gathered}
$$

\section{Appendix B}

The presentation of the physical significance of the quantities $t\left(k_{1}, k_{2}, k_{3}\right)$ and $r\left(k_{1}, k_{2}, k_{3}\right)$ is to be started with the calculation of the probability-current-density function, $j(x)=\hbar\left[\varphi^{*}(x) \partial \varphi(x) / \partial x-\varphi(x) \partial \varphi^{*}(x) / \partial x\right]$ $/ 2 \mathrm{i} m$. On the left-hand side of the biased double delta-barrier, it is

$$
\begin{aligned}
& j_{\mathrm{I}}(x)=\left[1-r^{*}\left(k_{1}, k_{2}, k_{3}\right) r\left(k_{1}, k_{2}, k_{3}\right)\right] A_{\mathrm{I}}^{*} A_{\mathrm{I}} \frac{\hbar k_{1}}{m} \\
& \quad-t^{*}\left(k_{1}, k_{2}, k_{3}\right) t\left(k_{1}, k_{2}, k_{3}\right) B_{\mathrm{III}}^{*} B_{\mathrm{III}} \frac{\hbar k_{3}}{m} \\
& \quad-\left[r^{*}\left(k_{1}, k_{2}, k_{3}\right) t\left(k_{1}, k_{2}, k_{3}\right) A_{\mathrm{I}}^{*} B_{\mathrm{III}}\right. \\
& \left.\quad+t^{*}\left(k_{1}, k_{2}, k_{3}\right) r\left(k_{1}, k_{2}, k_{3}\right) B_{\mathrm{III}}^{*} A_{\mathrm{I}}\right] \frac{\hbar \sqrt{k_{1} k_{2}}}{m} .
\end{aligned}
$$

On the right-hand side of the biased double delta-barrier, it is

$$
\begin{aligned}
j_{\mathrm{III}} & (x)=t^{*}\left(k_{1}, k_{2}, k_{3}\right) t\left(k_{1}, k_{2}, k_{3}\right) A_{\mathrm{I}}^{*} A_{\mathrm{I}} \frac{\hbar k_{1}}{m} \\
- & {\left[1-r^{*}\left(k_{1}, k_{2}, k_{3}\right) r\left(k_{1}, k_{2}, k_{3}\right)\right] B_{\mathrm{III}}^{*} B_{\mathrm{III}} \frac{\hbar k_{3}}{m} } \\
- & {\left[r^{*}\left(k_{1}, k_{2}, k_{3}\right) t\left(k_{1}, k_{2}, k_{3}\right) A_{\mathrm{I}}^{*} B_{\mathrm{III}}\right.} \\
& \left.+t^{*}\left(k_{1}, k_{2}, k_{3}\right) r\left(k_{1}, k_{2}, k_{3}\right) B_{\mathrm{III}}^{*} A_{\mathrm{I}}\right] \frac{\hbar \sqrt{k_{1} k_{2}}}{m} .
\end{aligned}
$$

Evidently, the probability-current-density function $j(x)$ is constant, $j_{\mathrm{III}}(x)=j_{\mathrm{I}}(x)$. If $B_{\mathrm{III}}=0$, the wave function $\delta(x)$ consists of three terms. The first term, $A_{\mathrm{I}} \mathrm{e}^{+\mathrm{i} k_{1} x}$, represents a plane wave that is incident on the biased double delta-barrier from $-\infty$. The interaction with the biased double delta-barrier produces a reflected plane wave, $r\left(k_{1}, k_{2}, k_{3}\right) A_{\mathrm{I}} \mathrm{e}^{-\mathrm{i} k_{1} x}$, which escapes to $-\infty$, and a transmitted plane wave, $\sqrt{k_{1} / k_{3}} t\left(k_{1}, k_{2}, k_{3}\right) A_{\mathrm{I}} \mathrm{e}^{+\mathrm{i} k_{3} x}$, which moves off to $+\infty$. It is seen from the above equation that the incident flux, the reflected flux, and the transmitted flux are $A_{\mathrm{I}}^{*} A_{\mathrm{I}} \hbar k_{1} / m, \quad r^{*}\left(k_{1}, k_{2}, k_{3}\right) r\left(k_{1}, k_{2}, k_{3}\right) A_{\mathrm{I}}^{*} A_{\mathrm{I}} \hbar k_{1} / m$ and $t^{*}\left(k_{1}, k_{2}, k_{3}\right) t\left(k_{1}, k_{2}, k_{3}\right) A_{\mathrm{I}}^{*} A_{\mathrm{I}} \hbar k_{1} / m$, respectively. Thus, the quantities $t\left(k_{1}, k_{2}\right)$ and $r\left(k_{1}, k_{2}\right)$ give the transmission amplitude and the reflection amplitude, respectively. The transmission and reflection coefficient are given by $T\left(k_{1}, k_{2}, k_{3}\right)=t^{*}\left(k_{1}, k_{2}, k_{3}\right) t\left(k_{1}, k_{2}, k_{3}\right)$ and $R\left(k_{1}, k_{2}, k_{3}\right)=r^{*}\left(k_{1}, k_{2}, k_{3}\right) r\left(k_{1}, k_{2}, k_{3}\right)$, respectively. If $A_{\mathrm{I}}=0$, the wave function $\varphi(x)$ can be interpreted in a similar fashion. Its three terms represent a plane wave, which is incident on the biased double delta-barrier from $+\infty$, a reflected plane wave, which escapes to $+\infty$, and a transmitted plane wave, which moves off to $-\infty$.

\section{References}

[1] J. Peng, H. Chen, S. Zhou, J. Phys., Condens. Matter 1, 5451 (1989).

[2] Y. Zebda, A.M. Kan'an, J. Appl. Phys. 72, 559 (1992).

[3] B. Méndez, F. Dominquez-Adame, Am. J. Phys. 62, 143 (1994).

[4] J.M. Ziman, Principles of the Theory of Solids, University Press, Cambridge 1972, Ch. 6.

[5] D. Bohm, Quantum Theory, Prentice-Hall, New York 1952, Ch. 11.

[6] L.D. Landau, E.M. Lifshitz, Quantum Mechanics, Pergamon Press, Oxford 1977, Ch. 3.

[7] E. Merzbacher, Phys. Today 55, 44 (2002).

[8] S. Flügge, Practical Quantum Mechanics I, Springer-Verlag, Berlin 1974, Ch. 2.

[9] A. Galindo, P. Pascual, Quantum Mechanics I, Springer-Verlag, Berlin 1990, Ch. 4.

[10] V. Bezák, J. Math. Phys. 37, 5939 (1996).

[11] I. Yanetka, Phys. Status Solidi B 203, 363 (1997).

[12] S. Vatannia, G. Gildenblat, IEEE J. Quantum Electron. 32, 1093 (1996).

[13] I. Yanetka, Acta Phys. Univ. Comenianae XLVI-XLVII, 7 (2005-2006).

[14] I. Yanetka, Physica B 270, 371 (1999). 